\title{
Effects of serum homocysteine and adiponectin levels on platelet aggregation in untreated patients with essential hypertension
}

\author{
Hakan Ekmekçi · Özlem Balcı Ekmekçi · Serap Erdine · Hüseyin Sönmez • \\ Yusup Ataev · Zeynep Öztürk · İlknur İşler Bütün · Çiğdem Gürel · \\ Mine Kucur · Nurver Turfaner · Turgut Ulutin · Sevim Purisa • \\ Vural Ali Vural
}

Published online: 28 August 2009

(C) Springer Science+Business Media, LLC 2009

\section{Erratum to: J Thromb Thrombolysis \\ DOI 10.1007/s11239-008-0292-0}

The author regrets an error occurred in the Table 2 of this article. The unit of adiponectin (APN) should be $(\mu \mathrm{g} / \mathrm{ml})$ instead of $\mathrm{ng} / \mathrm{ml}$. The corrected table is given below.

The online version of the original article can be found under doi:10.1007/s11239-008-0292-0.

H. Ekmekçi · Ö. B. Ekmekçi

Istanbul Medical Faculty, Bone Marrow Transplantation Unit, Department of Pediatric Haematology and Oncology,

Istanbul University, Istanbul, Turkey

H. Ekmekçi ( $₫)$

Seyit Omer Mah. Altimermer cad., Yeni yol sok. Saray Ap. A Blok. No:5/17, 34290 Istanbul, Kocamustafapasa, Turkey

e-mail: hakekmekci@yahoo.com

S. Erdine $\cdot$ Y. Ataev · V. A. Vural

Cerrahpasa Medical Faculty, Department of Cardiology,

Istanbul University, Istanbul, Turkey

H. Sönmez · Z. Öztürk · İ. İ. Bütün · M. Kucur

Cerrahpasa Medical Faculty, Department of Biochemistry, Istanbul University, Istanbul, Turkey

Ç. Gürel · T. Ulutin

Cerrahpasa Medical Faculty, Department of Medical Biology,

Istanbul University, Istanbul, Turkey

\section{N. Turfaner}

Cerrahpasa Medical Faculty, Department of Family Medicine, Istanbul University, Istanbul, Turkey

S. Purisa

Cerrahpasa Medical Faculty, Department of Biostatistics,

Istanbul University, Istanbul, Turkey
Table 2 ADP-induced platelet aggregation, adiponectin, homocysteine, $\mathrm{TNF}-\alpha$, hsCRP, NOx, nitrite and nitrate levels in hypertensive patients and controls

\begin{tabular}{lccr}
\hline & $\begin{array}{l}\text { Controls } \\
(n=13)\end{array}$ & $\begin{array}{l}\text { Hypertensive } \\
\text { patients }(n=23)\end{array}$ & \multicolumn{1}{l}{$P$} \\
\hline ADP-S $(\mathrm{ohms})$ & $118.3 \pm 38.1$ & $127.9 \pm 42.5$ & 0.490 \\
ADP-A $(\%)$ & $84.8 \pm 21.3$ & $93.7 \pm 14.3$ & 0.140 \\
APN $(\mu \mathrm{g} / \mathrm{ml})$ & $57.3 \pm 10.4$ & $37.7 \pm 16.4$ & $<0.001$ \\
Hcy $(\mu \mathrm{mol} / \mathrm{l})$ & $9.4 \pm 2.8$ & $12.6 \pm 4.2$ & 0.020 \\
TNF- $\alpha(\mathrm{pg} / \mathrm{ml})$ & $23.5 \pm 6.9$ & $30.4 \pm 18.2$ & 0.190 \\
hsCRP $(\mathrm{mg} / \mathrm{l})$ & $1.7 \pm 0.6$ & $3.3 \pm 2.0$ & 0.001 \\
NOx $(\mu \mathrm{M})$ & $15.8 \pm 4.7$ & $14.4 \pm 4.3$ & 0.370 \\
Nitrite $(\mu \mathrm{M})$ & $4.6 \pm 2.3$ & $3.3 \pm 1.8$ & 0.045 \\
Nitrate $(\mu \mathrm{M})$ & $11.3 \pm 3.8$ & $11.1 \pm 3.8$ & 0.950 \\
\hline
\end{tabular}

ADP-S, ADP-induced platelet aggregation slope; ADP-A, ADPinduced platelet aggregation amplitude; APN, adiponectin; Hcy, homocysteine; TNF- $\alpha$, tumor necrosis factor-alpha; hsCRP, high sensitive C-reactive protein; NOx, total nitric oxide 